\title{
Effects of Flow Profile on Educed Acoustic Liner Impedance
}

\author{
M. G. Jones*, W. R. Watson ${ }^{\dagger} a n d$ D. M. Nark ${ }^{\ddagger}$
}

\begin{abstract}
This paper presents results of an investigation of the effects of shear flow profile on impedance eduction processes employed at NASA Langley. Uniform and 1-D shear-flow propagation models are used to educe the acoustic impedance of three test liners based on aeroacoustic data acquired in the Langley Grazing Flow Impedance Tube, at source levels of 130, 140 and $150 \mathrm{~dB}$, and at centerline Mach numbers of $0.0,0.3$ and 0.5 . A ceramic tubular, calibration liner is used to evaluate the propagation models, as this liner is expected to be insensitive to SPL, grazing flow Mach number, and flow profile effects. The propagation models are then used to investigate the effects of shear flow profile on acoustic impedances educed for two conventional perforate-over-honeycomb liners. Results achieved with the uniform-flow models follow expected trends, but those educed with the 1-D shear-flow model do not, even for the calibration liner. However, when the flow profile used with the shear-flow model is varied to increase the Mach number gradient near the wall, results computed with the shear-flow model are well matched to those achieved with the uniform-flow model. This indicates the effects of flow profile on educed acoustic liner impedance are small, but more detailed investigations of the flow field throughout the duct are needed to better understand these effects.
\end{abstract}

\section{Introduction}

While the total volume of commercial air traffic is expected to approximately double over the next 15 years, aircraft noise constraints continue to become increasingly stringent. As part of NASA's Fundamental Aeronautics Program, focused efforts are currently underway to develop concepts to confine objectionable aircraft noise within the boundaries of typical airports. Acoustic liners mounted in the aircraft engine nacelles provide a significant portion of the current fan-noise reduction, but they must be further optimized if this challenging goal is to be achieved. This will require more detailed understanding of acoustic impedance, an intrinsic acoustic parameter for liners that is dependent on the source sound pressure level and the grazing flow profile at the liner surface.

Many researchers have studied the effects of shear flow profiles on the sound attenuation achieved with conventional liners. In 1958, Pridmore-Brown ${ }^{1}$ conducted investigations of the propagation of sound above a flat surface in both a uniform flow and a turbulent shear flow. He observed that the flow gradient tends to channel the sound energy into a narrow layer next to the wall for downstream propagation. Tack et al. ${ }^{2}$ conducted theoretical and experimental investigations of the influence of shear flow on the attenuation of sound in a lined duct, for upstream and downstream sound propagation. They observed that a uniform-flow assumption is adequate for low-frequency propagation, but were unable to accurately predict high-frequency attenuation with either uniform or shear-flow models. In fact, they found the predicted attenuation to be nearly independent of the flow profile for higher frequencies. They postulated that this was due to a failure to carry a sufficient number of terms in their solution. In the early 1970's, Eversman ${ }^{3}$ conducted $^{2}$ numerical studies that indicated refraction of sound through a shear flow in an inlet (away from the walls of the duct) results in reduction of achievable attenuation of the least attenuated mode, especially at higher Mach numbers. However, he found the effects of shear flow to be greatly reduced for exhaust flow. More

* Senior Research Scientist, Research Directorate, Structural Acoustics Branch, AIAA Associate Fellow.

${ }^{\dagger}$ Senior Research Scientist, Research Directorate, Computational AeroSciences Branch, AIAA Associate Fellow.

${ }^{\ddagger}$ Research Scientist, Research Directorate, Structural Acoustics Branch, AIAA Member. 
recently, Li et al. ${ }^{4}$ used a time-domain analysis to further evaluate the effects of mean flow profile shape. They found similar results to those reported earlier, but also found that the mean flow profile may excite flow instabilities. Based on these earlier investigations, it seems reasonable to assume uniform-flow propagation codes can be successfully used to educe acoustic liner impedance for downstream propagation, but shear-flow propagation codes are needed for upstream propagation.

For over 30 years, NASA Langley has invested significant effort in the development of numerical prediction tools and experimental rigs for the evaluation of acoustic liners. This has included the development of numerical prediction tools to account for the effects of sheared flows through a flow duct. In 2001, Watson et $a l .{ }^{5}$ presented a method for educing acoustic liner impedance in the presence of grazing, parallel shear flow. The key features of the method were a numerical solution to the linearized frequency-domain equations of mass, momentum, and energy conservation, and the minimization of an objective function. Based on acoustic pressure measurements in the NASA Langley Grazing Incidence Tube (GIT), the impedance spectra of two dissimilar liners were educed in the presence of a downstream, parallel, mean shear flow. Although the mean flow propagation was downstream, the importance of accurately modeling the effects of mean shear to educe liner impedance was clearly demonstrated. On the surface, this appears to be in direct contrast to the earlier studies mentioned above, where the importance of including shear flow effects in the analysis was minimal for downstream propagation. However, there is one key difference. The goal of the "impedance eduction process" is to determine the impedance of the test liner, regardless of the aeroacoustic environment to which the liner is exposed. Since the shear flow does not have a significant effect on the amount of absorption for a given liner, the process of educing a correct impedance for the liner becomes more difficult in the presence of this shear flow, as smaller effects are harder to distinguish. In spite of this increased difficulty, it is critical that the correct impedance be educed, as it is this impedance that will be used as a boundary condition for aeroacoustic propagation codes to predict the liner absorption to be achieved in aircraft engine nacelles. Hence, as the requirements for optimized acoustic liners become increasingly demanding, inclusion of the shear flow effects for both upstream and downstream propagation are likely to become even more important.

Regardless, the vast majority of results presented thus far ${ }^{6-11}$ have been based on a uniform-flow assumption, the use of which greatly simplifies the analysis for a number of reasons. Perhaps the most important is the fact that horizontal and vertical spanwise modes are uncoupled in rigid-walled ducts. In addition, the acquisition of high quality flow profile data requires detailed measurements and is therefore time-consuming. Finally, the previous flow ducts used for acoustic liner evaluation were not designed for convenient modification of the flow profile. Efforts to use flow injection and boundary layer suction to modify the boundary layer in a controlled manner have provided modest success, ${ }^{12}$ but were quite cumbersome to implement.

The Langley Grazing Flow Incidence Tube has been used by researchers throughout the liner community for the evaluation of acoustic propagation codes and for investigations of the effects of different parameters on the acoustic characteristics of liners. Lessons learned from the previous three decades of research were applied to the design of a replacement flow duct, labeled the Grazing Flow Impedance Tube (GFIT). A few of these design features were of particular value to the current study. A modular design was implemented such that portions of the duct can be easily interchanged, thereby allowing movement of the test window to multiple axial locations. Also, a dual-axis traverse probe system was implemented for efficient flow profile measurement.

The purpose of the current study is to investigate the effects of shear flow profile on the impedance eduction process employed at NASA Langley. First, a linear, calibration liner is used to evaluate the process. This liner has been previously demonstrated ${ }^{8}$ to be insensitive to grazing flow Mach number (i.e., average Mach number), and is expected to also be insensitive to flow profile effects. Next, two conventional, nonlinear liners, each constructed with a perforate facesheet bonded to a honeycomb core and rigid backplate, are considered. These liners have been previously demonstrated ${ }^{13}$ to be sensitive to grazing flow Mach number (resistance increases with increasing Mach number). The effects of mean flow profile are also expected to be present for these liners, although the earlier investigations mentioned above would suggest that these effects should be minimal, as downstream propagation is present for this test. A dual-axis traverse probe system is used to acquire flow profile data at multiple axial locations in the GFIT, such that the effects of naturally developing boundary layer flow can be assessed. The test window is then moved to allow acquisition of the acoustic pressure data used in the impedance eduction process while the liner is exposed to the desired mean flow profile. These data are processed with three propagation models, one based on the convected Helmholtz equation, and two based on the Pridmore-Brown equation (uniform and 1-D shear 
flow versions). A description of the acoustic and mean flow analyses is provided in Section II. Section III provides a description of the three test samples, and Section IV provides a description of the experimental methods used in this investigation. Results of the investigation are provided in Section V, and concluding remarks regarding some of the more signicant results are provided in Section VI.

\section{Aeroacoustic Propagation Models}

Two propagation models are used in the current investigation. The first uses a finite element method to solve for the acoustic pressure field in a rectangular duct containing a finite-length acoustic liner with known impedance and uniform mean flow. The second propagation model assumes the duct is infinitely long, with the upper wall consisting of a uniform-impedance liner, and allows for either uniform or 1-D shear flow.

\section{Model 1 - Finite Length Liner}

This model assumes the surface of the finite-length, test liner forms a portion of the upper wall of the rectangular duct, and the remainder of the duct consists of rigid walls. It also assumes the mean flow to be uniform (i.e., plug flow). The analysis used in this model has been presented in a previous paper, ${ }^{14}$ and is summarized here for convenience. A schematic of the three-dimensional flow duct and Cartesian coordinate system is provided in figure 1. The lower and two side walls of the duct are rigid, and the source and exit planes of the computational domain are located at $x=0$ and $x=L$, respectively. The upper wall is also rigid, except for the portion between $L_{1} \leq x \leq L_{2}$, which contains the test liner with an unknown, uniform normalized impedance, $\zeta$. The sound source is assumed to be a single tone at a frequency such that no higher-order spanwise (in the $z$-direction) modes propagate, thereby allowing the analysis to be restricted to the $(x, y)$-plane.

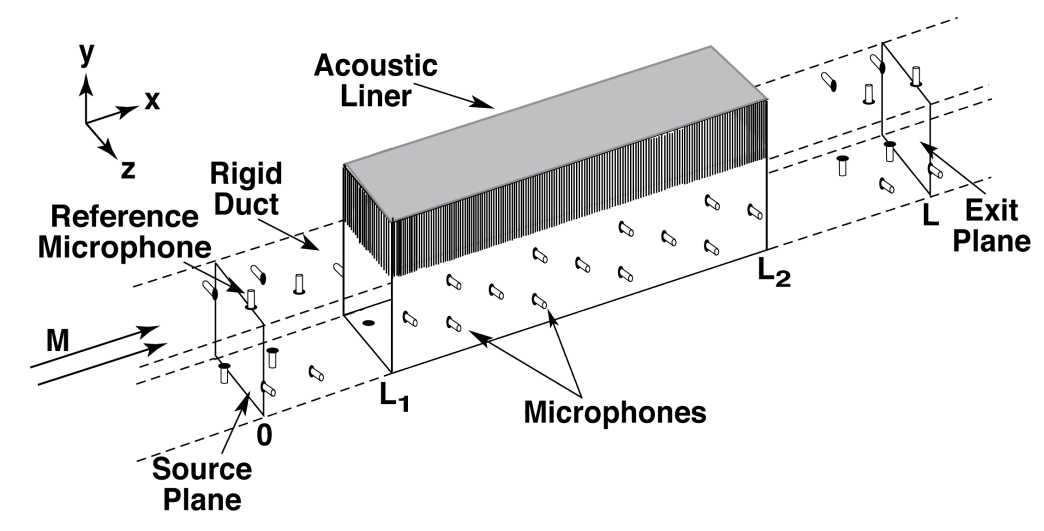

Figure 1: Sketch of Grazing Flow Impedance Tube computational domain.

The acoustic pressure field, $p$, propagating through the duct under the assumed conditions satisfies the convected Helmholtz equation ${ }^{15}$

$$
\left(1-M^{2}\right) \frac{\partial^{2} p}{\partial x^{2}}+\frac{\partial^{2} p}{\partial y^{2}}-2 i k M \frac{\partial p}{\partial x}+k^{2} p=0
$$

where $k=\omega / c$ is the freespace wavenumber, $M=U / c$ is the mean flow Mach number, $U$ is the uniform axial flow speed, and $c$ is the sound speed in the duct.

The wall impedance boundary condition for the locally-reacting liner has been discussed in detail by Myers. ${ }^{16}$ For the upper wall, this condition is

$$
-\frac{\partial p}{\partial y}=i k\left(\frac{p}{\zeta}\right)+2 M \frac{\partial}{\partial x}\left(\frac{p}{\zeta}\right)+\frac{M^{2}}{i k} \frac{\partial^{2}}{\partial x^{2}}\left(\frac{p}{\zeta}\right)
$$


where $\zeta$ represents the impedance of the upper wall. The admittance (i.e., $1 / \zeta$ ) is taken as zero for rigid upper wall sections of the duct located upstream and downstream of the liner. The wall impedance boundary condition of the rigid lower wall is

$$
\frac{\partial p}{\partial y}=0
$$

An array of microphones flush-mounted in the hardwall section upstream of the test liner is used to determine the acoustic pressure profile, $p_{s}(y)$, at the source plane. This acoustic pressure profile is used as the source plane boundary condition

$$
p(0, y)=p_{s}(y)
$$

A similar microphone array in the hardwall section downstream of the test liner is used to determine the exit plane acoustic pressure boundary condition, $p_{e}(y)$

$$
p(L, y)=p_{e}(y)
$$

Note that the acoustic pressure measurements must be obtained with the test liner installed and must contain the effects of higher-order modes in the vertical direction (i.e., the $y$ direction). Equations (1)-(5) do not have exact solutions for a general set of input parameters. Consequently, they are solved numerically using a finite element method.

An essential feature of the impedance eduction technique employed in this paper is the development of a general purpose numerical procedure to determine the impedance that reproduces the acoustic pressure field measured on the wall opposite the test liner. The wall error function

$$
F(\zeta)=\sum_{I=1}^{n w a l l}\left\{\left.p\left(x_{I}, 0\right)\right|_{\mathrm{FEM}}-\left.p\left(x_{I}, 0\right)\right|_{\text {Meas }}\right\}\left\{\left.p^{*}\left(x_{I}, 0\right)\right|_{\mathrm{FEM}}-\left.p^{*}\left(x_{I}, 0\right)\right|_{\text {Meas }}\right\}
$$

is used as an objective function, and the unknown impedance is selected such that this objective function is minimized. The above objective function may be interpreted as the difference between the measured and computed wall pressures opposite the test liner. Note that the proposed procedure is perfectly general and requires only that the formulation of the propagation model constitute a well-posed boundary value problem. Thus, nonuniform impedance and flows containing shear layers are possible within the context of the current impedance eduction theory provided that the governing Eq. (1) is modified to account for the shear layer. For convenience, this model is referenced as CHE-FE (convected Helmholtz equation, finite-element method) in the following discussion.

\section{Model 2 - Infinite Length Liner}

The second propagation model assumes the upper wall of an infinitely long duct contains an acoustic liner with uniform impedance. This model computes the impedance of the liner by numerically solving the Pridmore-Brown ${ }^{1}$ second-order ordinary differential equation, given as

$$
\frac{d^{2} P}{d y^{2}}+\frac{2 k_{x}}{\left(k-M k_{x}\right)} \frac{d M}{d y} \frac{d P}{d y}+\left[k^{2}-2 k k_{x} M-\left(1-M^{2}\right) k_{x}^{2}\right] P=0
$$

where the 1-D acoustic pressure $P(y)$ is provided by the relation

$$
p=P(y) e^{-i k_{x} x}
$$

This equation is solved as a two-point boundary value problem, with the boundary conditions at the lower wall given as

$$
P(0)=1 \quad \text { and }\left.\quad \frac{d P}{d y}\right|_{y=0}=0
$$


To obtain the solution to the Pridmore-Brown equation, the axial propagation constant, $k_{x}$, is computed from a measurement of the sound pressure level and phase decay rates, $d \mathrm{SPL} / d x$ and $d \phi / d x$, using the equation:

$$
k_{x}=-\frac{d \phi}{d x}+\frac{d \mathrm{SPL}}{d x} \frac{i}{20 \log _{10}(e)}
$$

This assumes that the axial sound pressure level and phase decay rates can be determined from the acoustic pressures measured on the wall opposite the liner (at $y=0$ ), and that they are negative for right-moving waves. It also assumes that this acoustic pressure profile is dominated by a single mode.

Clearly, the acoustic liner is not infinitely long, as assumed in this model. Instead, it extends over a finite length ( $L_{1} \leq x \leq L_{2}$ in Fig. 1). Thus, for the current investigation, a portion of the acoustic pressure profile (as measured along the lower wall opposite the liner) that extends over the $304.8 \mathrm{~mm}$ centered portion of the liner $\left(L_{1}+51.8 \mathrm{~mm}<x<L_{2}-51.8 \mathrm{~mm}\right)$ is used to determine the SPL and phase decay rates. This axial range was selected to avoid the effects of higher-order modes generated by the impedance discontinuities at the liner leading and trailing edges, and to minimize the effects of standing waves set up by these same impedance discontinuities. The selected portion of the acoustic pressure profile is curve-fit (linear slope) to compute the required decay rates, and correlation coefficients are computed for these curve-fits to form the basis for determining whether a single-order mode is dominant over the axial extent of interest. For those test conditions where the correlation coefficients are sufficiently high to suggest the acoustic pressure profile is dominated by a single-order mode (i.e., SPL and $\phi$ profiles decay with constant slopes), the surface impedance of the acoustic liner is computed as

$$
\zeta=-i k P\left(1-\frac{k_{x}}{k} M\right)^{2}\left(\frac{d P}{d y}\right)^{-1} \quad \text { at } y=H
$$

where $H$ is the duct height (the liner surface is located at $y=H$ ). This model holds for uniform flow $(M(y)=$ constant $)$ or 1-D shear flow $(M(y) \neq$ constant). For convenience, the uniform and shear-flow versions of this model will be referenced as PBU-SM (Pridmore-Brown, uniform flow, single-mode method) and PBS-SM (Pridmore-Brown, 1-D shear flow, single-mode method), respectively.

\section{Impedance Eduction Processes}

Each of the two propagation models described above are used with eduction processes to determine the acoustic impedance spectrum of the test liner being evaluated. For each test condition of interest (selected SPL and M), acoustic pressures are acquired at each microphone along the lower wall over the axial range of $0 \leq x \leq L$. Mean flow data are also acquired at axial planes just upstream and downstream of the acoustic liner. These data are used to compute average Mach numbers for use in the uniform-flow models (CHE-FM and PBU-SM), and 1-D shear flow profiles for the shear-flow model (PBS-SM).

The CHE-FM propagation model uses these mean flow and acoustic data to determine the test liner impedance via the following iterative process:

1. An initial value of impedance, $\zeta$, is assumed for the liner.

2. Equations (1-5) are used to compute the acoustic pressures at the lower wall microphone locations via a finite element method.

3. These computed acoustic pressures are compared with the measured acoustic pressures to compute an error function [Equation (6)].

4. If the error function is sufficiently small (within a predetermined tolerance), the process is assumed to have converged, and the impedance selected above is taken to be the impedance of the liner.

5. If the error function is too large, the impedance is varied and the process is repeated. This continues until the process converges. This iterative process is controlled via an optimization routine. 


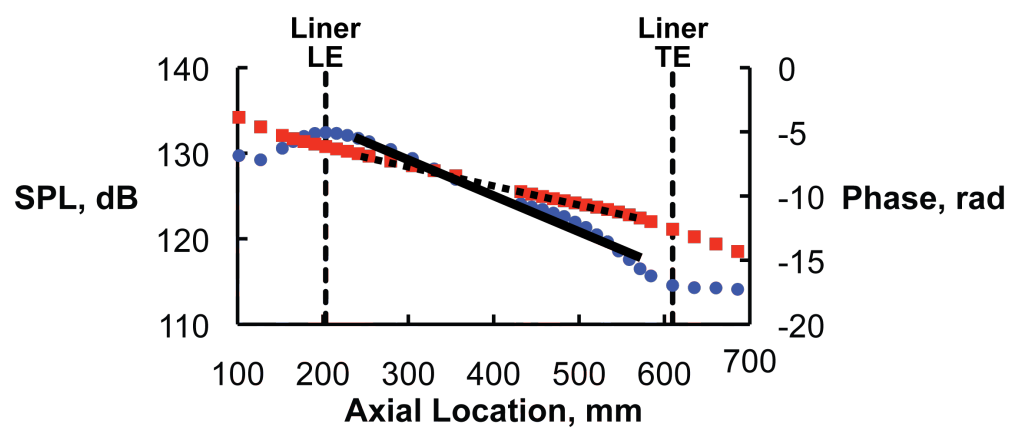

Figure 2: Sample acoustic pressure profile; Red squares - SPL; blue circles - Phase; solid line - linear fit of $\mathrm{SPL}(\mathrm{x})$; dashed line - linear fit of Phase(x).

The PBU-SM and PBS-SM propagation models use these same mean flow data, but only use a portion of the measured acoustic pressures. These models assume the liner is of infinite extent, which implies the absence of reflections, as there are no impedance discontinuities in the upper wall containing the infinitelength liner (the liner is assumed to provide uniform impedance over its entire surface). This is clearly not the case in the GFIT. Instead, reflections occur at the leading and trailing edges of the liner. For this reason, only the portion of data away from the liner edges (for the current study, this distance is taken to be at least $51 \mathrm{~mm}$ ) is considered for use in this model (i.e., over the axial range of $L_{1}+51 \mathrm{~mm} \leq x \leq L_{2}-51 \mathrm{~mm}$ ). These propagation models are used as follows to determine the test liner impedance:

1. The acoustic pressure profile over the axial range of $L_{1}+51 \mathrm{~mm} \leq x \leq L_{2}-51 \mathrm{~mm}$ is evaluated to determine whether the SPL and phase profiles decay monotonically (e.g., as in Fig. 2). If both decay monotonically, the assumption that the sound field is "dominated" by a single mode is satisfied. If one or both do not decay monotonically, the single-mode assumption is violated and these propagation models (PBU-SM and PBS-SM) cannot be used. It should be noted that this requirement is evaluated independently at each test frequency of interest.

2. For those test frequencies where the SPL and phase profiles decay monotonically, Equation (10) is used to compute the axial wavenumber, $k_{x}$, from the SPL and phase decay rates.

3. The two-point boundary value problem defined by Equations (7-9) is solved numerically, and Equation (11) is used to compute the impedance of the test liner.

This process allows a direct solution for the test liner impedance, and is therefore less time-consuming than the finite element method described above. However, the authors prefer to use the finite element method where possible, as it is fully automated and can also handle the effects of higher-order modes in the portion of the duct containing the liner.

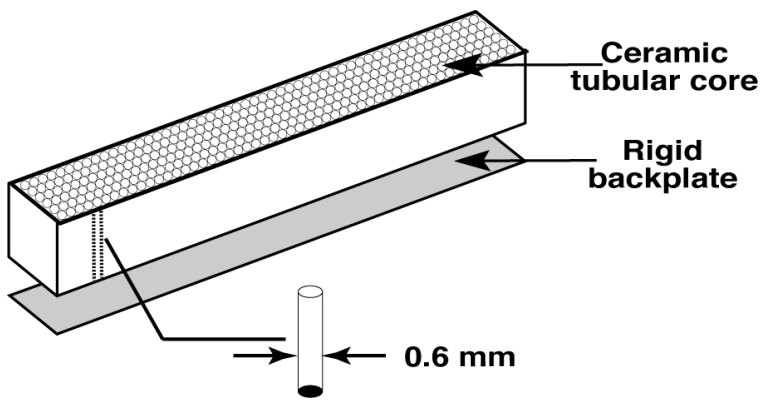

Figure 3: Sketch of ceramic tubular liner. 


\section{Test Samples}

Three acoustic liners are used in the current investigation. The first is a ceramic tubular liner that has been studied extensively over the last 15 years. ${ }^{6,7,17}$ This liner has been demonstrated to be nearly linear with respect to SPL and grazing flow Mach number, and is thus expected to also be insensitive to flow profile effects. The other two liners are conventional, perforate-over-honeycomb liners. The effects of mean flow profile are expected to be more significant for these liners, although the earlier investigations mentioned above would suggest that these effects should be minimal, as downstream propagation is used for this test.

\section{Ceramic Tubular Liner}

The ceramic tubular liner (Fig. 3) consists of parallel, cylindrical channels embedded in a ceramic matrix. These channels, with diameters of $0.6 \mathrm{~mm}$, are perpendicular to the exposed surface and provide a surface porosity of $57 \%$. The $85.6 \mathrm{~mm}$-deep channels are rigidly terminated such that each is isolated from its neighbor to ensure a locally-reacting structure. The channel diameter is small enough that SPL and grazing flow effects are insignificant relative to internal viscous losses. This liner, labeled as CT57 in the remainder of this paper, provides an impedance spectrum that varies over a range typically observed in aircraft engine nacelle liners.

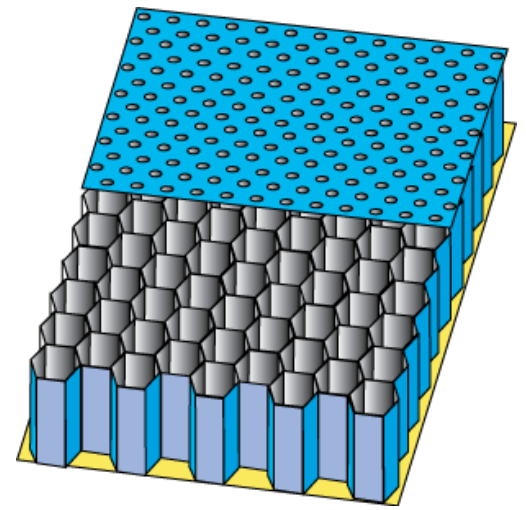

Figure 4: Sketch of conventional liner.

\section{Conventional Liners}

Two conventional liners are used in this investigation. Each consists of a single-layer, perforate-overhoneycomb liner geometry (Fig. 4) representative of liners commonly used in current aircraft engine nacelles. Each liner has a facesheet with $0.991 \mathrm{~mm}$-diameter holes and a thickness of $0.635 \mathrm{~mm}$, which is bonded to a $38.18 \mathrm{~mm}$-deep cavity consisting of $9.5 \mathrm{~mm}$-diameter hexcel honeycomb core, and is terminated with a rigid backplate. The surface porosities of these liners (labeled as GE01 and GE04, respectively) are 8.7\% and $13.2 \%$. These liners are expected be be nonlinear with respect to mean flow velocity and SPL. It is expected that the resistance spectra educed for these liners will increase as the mean flow Mach number is increased, but the choice of uniform or shear flow in the model will have limited effect.

\section{Experimental Methods}

\section{Grazing Flow Impedance Tube}

The Grazing Flow Impedance Tube (GFIT) has a cross-sectional geometry of $51 \mathrm{~mm}$ by $63 \mathrm{~mm}$, such that higher-order modes in the horizontal and vertical dimensions cut on at different frequencies. It allows convenient evaluation of acoustic liners with lengths from $51 \mathrm{~mm}$ to $610 \mathrm{~mm}$, in increments of $51 \mathrm{~mm}$. As depicted in Fig. 5, flow propagates from left to right. High pressure air is supplied on the upstream end, and a vacuum blower is located on the downstream end, such that the test window containing the acoustic liner is exposed to near-ambient pressure conditions for mean flows up to Mach 0.6. The surface of the test 
liner forms a portion (406 $\mathrm{mm}$ for the current investigation) of the upper wall of the flow duct. Eighteen acoustic drivers are mounted upstream of the test window, and are used to generate tones (one frequency at a time) at up to $150 \mathrm{~dB}$ over a frequency range of 0.4 to $3.0 \mathrm{kHz}$. Ninety-five flush-mounted microphones are used to measure the acoustic pressure field over the axial extent of $0 \leq x \leq L$, such that the aforementioned techniques can be used to determine the impedance of the test liner.

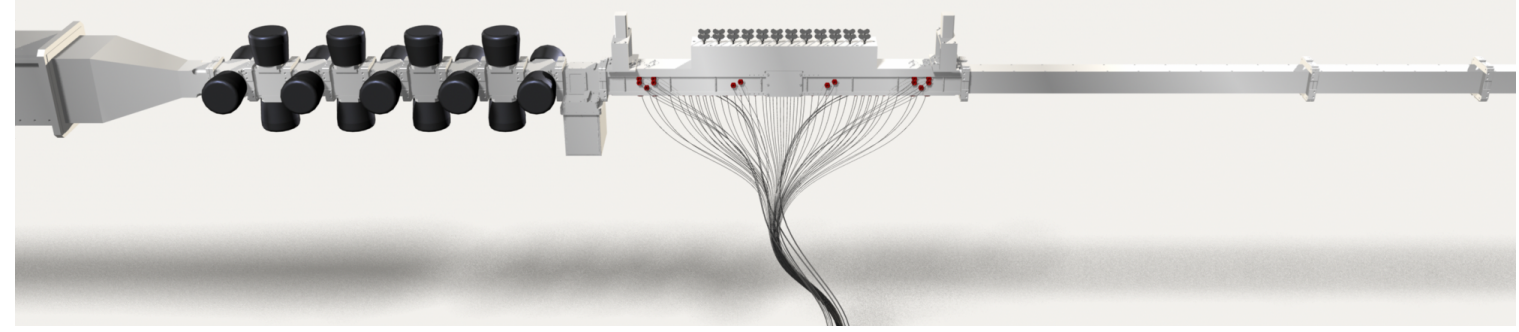

Figure 5: Sketch of Grazing Flow Impedance Tube.

\section{Flow Profile Measurements}

A dual-axis traverse probe system is used to measure the flow profile at selected axial planes in the GFIT. Two pitot probes are successively used for this measurement. One is shaped to allow detailed measurements close to the upper wall, and the other is shaped to allow corresponding measurements near the lower wall. The traverse system uses two stepping motors to successively move the pitot probe to preselected measurement locations, such that the full flow profile can be accurately determined. Due to the modular nature of the GFIT, it is relatively simple to move the traverse system to any desired axial location.

Figure 6 provides diagrams of the four duct configurations used in the current investigation. These configurations were chosen to (1) achieve distinct flow profiles, and (2) assess the effects of these different flow profiles on the educed acoustic impedance spectra of three test liners. For each configuration, the pitot-static probe positioned in section $\mathrm{E}$ was used to measure the setpoint, centerline Mach number $(0.0$, 0.3 or 0.5 . Two pitot probes were sequentially placed in section $\mathrm{D}$, and the dual-axis traverse probe system was used to measure the total pressure profile at 29 vertical locations between the lower and upper walls along the spanwise centerline of the duct. This measured total pressure profile was combined with a static pressure measurement in the same axial plane to provide a Mach number profile to be used as input to the aeroacoustic propagation models described earlier.

These 1-D shear flow profiles were acquired for each of the four configurations (i.e., with the traverse probe located at four axial locations) with each test liner installed in the test section, such that any effects of the liners on the flow profile could be included in the analysis. In total, twenty-four 1-D shear flow profiles were acquired with three liners. Measurements were conducted upstream and downstream of each liner for two centerline Mach numbers (0.3 and 0.5) with the test section positioned as shown in Configurations 1 and 2 (Fig. 6), and were repeated with the test section moved downstream as depicted in Configurations 3 and 4. More detailed 2-D shear flow profiles (9 vertical sweeps, with 29 measurements per sweep) were also acquired with Configurations 2 and 3, with a hardwall insert installed in the test section. For these measurements, the dual-axis traverse probes were traversed across entire axial planes. Contour maps of these 2-D shear flow profiles provide insight into the boundary layer growth along the axial extent of the duct.

For the analysis described earlier, uniform and 1-D shear flow assumptions were used. For the current investigation, the Mach number profile was assumed to vary linearly between the locations upstream and downstream of the liner. These two Mach number profiles were used to determine an "average" profile located at the axial midpoint of the test liner. For the 1-D shear flow analysis, this profile was used directly. For the uniform flow analysis, an average Mach number was computed from a curve-fit to the average flow profile. 


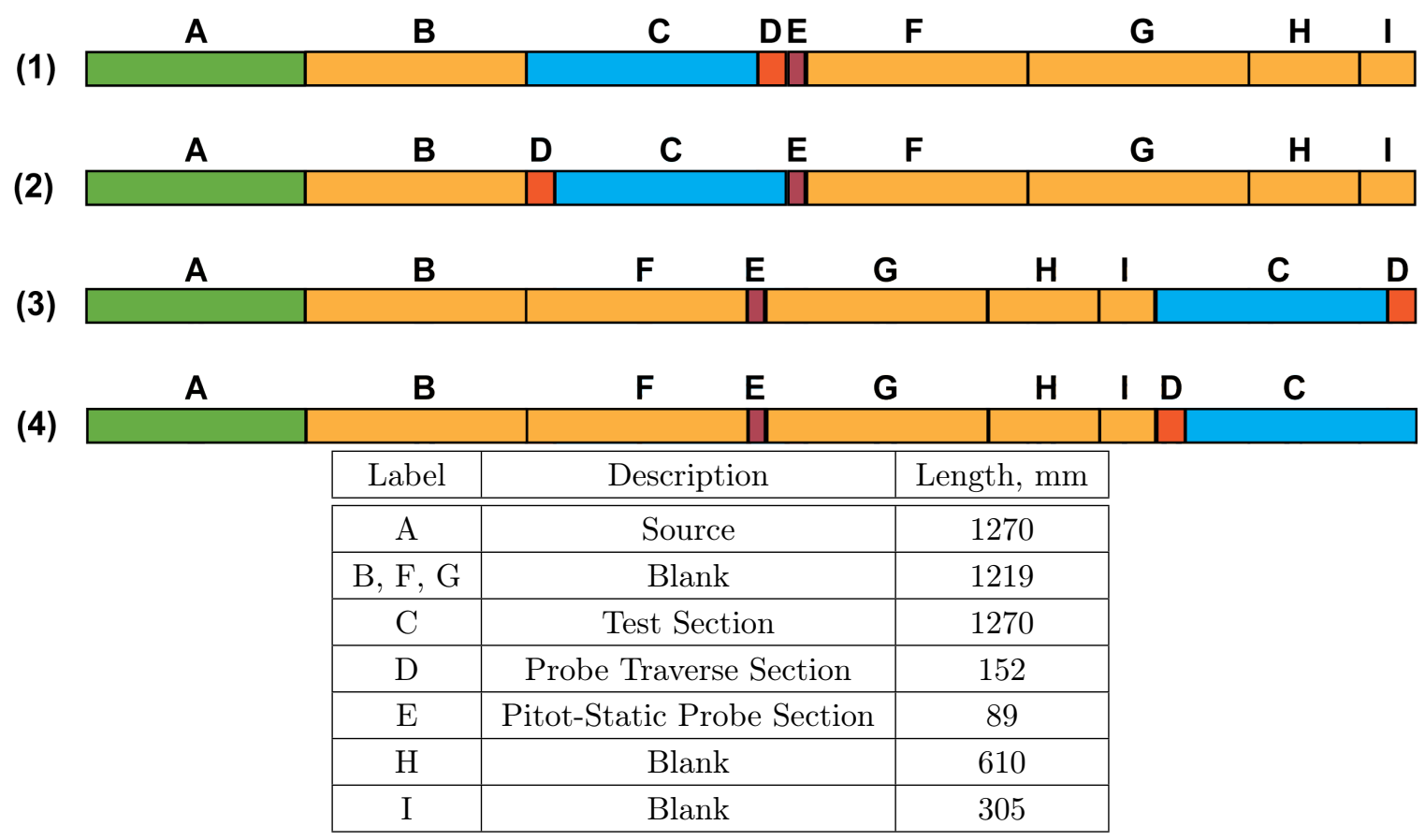

Figure 6: Configuration diagrams.

\section{Acoustic Measurements}

Ninety-five flush-mounted microphones are used to measure the complex acoustic pressure profile (amplitude and phase) in the vicinity of the acoustic liner. Analog-to-digital devices simultaneously acquire the data for 48 channels, and a switch matrix is used to switch from one set of 48 microphones (reference microphone plus microphones 2 through 48) to another set of 48 microphones (reference microphone plus microphones 49 through 95). The reference microphone (one of the 95 flush-mounted microphones, located in the source plane) provides the common basis for both sets of measurements, such that measurements at all 95 locations can be properly compared. All of this data is stored for more detailed evaluation as time permits. However, for the current investigation, only the 53 microphones located on the wall opposite the liner are used in the impedance eduction process. For each data acquisition, 2000 averages on each microphone channel (blocks of 2048 data points per average) are used. To reduce the influence of flow noise, a cross-spectrum signal extraction method ${ }^{18}$ is used to determine the amplitudes and phases at each of the microphone locations (relative to the amplitude and phase at the reference microphone location).

\section{Results}

\section{Mean Flow}

Figures 7 and 8 provide Mach number contour maps measured with the hardwall insert. In each figure, part (a) provides results acquired with the test section at the upstream end of the flow duct and the probe traverse section upstream of the test section (i.e., Configuration 2), whereas part (b) provides results acquired with the test section at the downstream end of the flow duct and the probe traverse section downstream of the test section (i.e., Configuration 3). A comparison of the these contour maps provides indications of the boundary layer growth over the length of the GFIT. The flow is fairly symmetric at the upstream location (Configuration 2), but the effects of corner flow are clearly evident at the downstream location (Configuration 3).

Table 1 contains pertinent flow profile parameters calculated from each of these contour plots. These parameters are calculated from the midspan, 1-D flow profiles (i.e., from $M(y)$ at $z=25.4 \mathrm{~mm}$ ). Along 


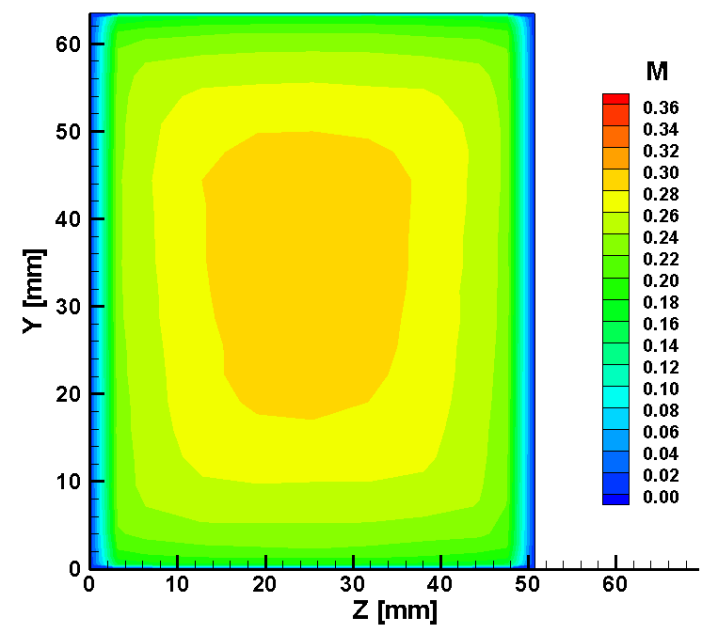

(a) Configuration 2

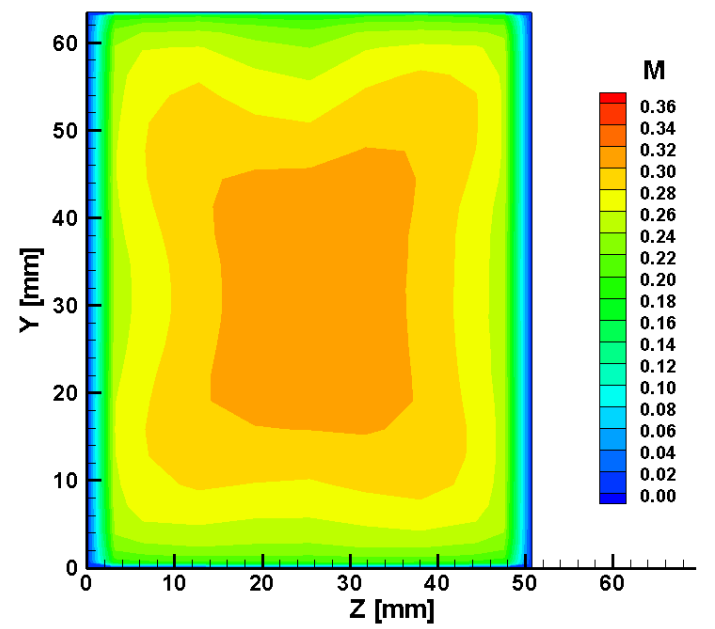

(b) Configuration 3

Figure 7: Mach number contour plots; $M_{\text {set }}=0.3$.

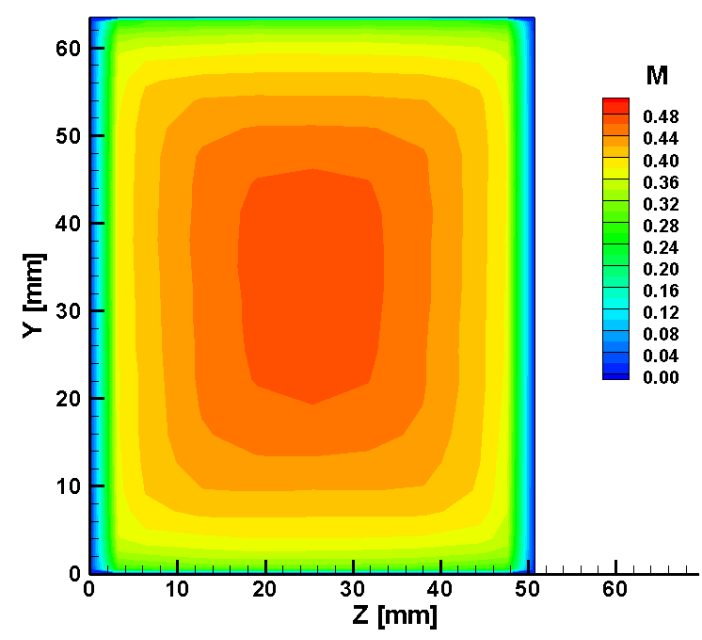

(a) Configuration 2

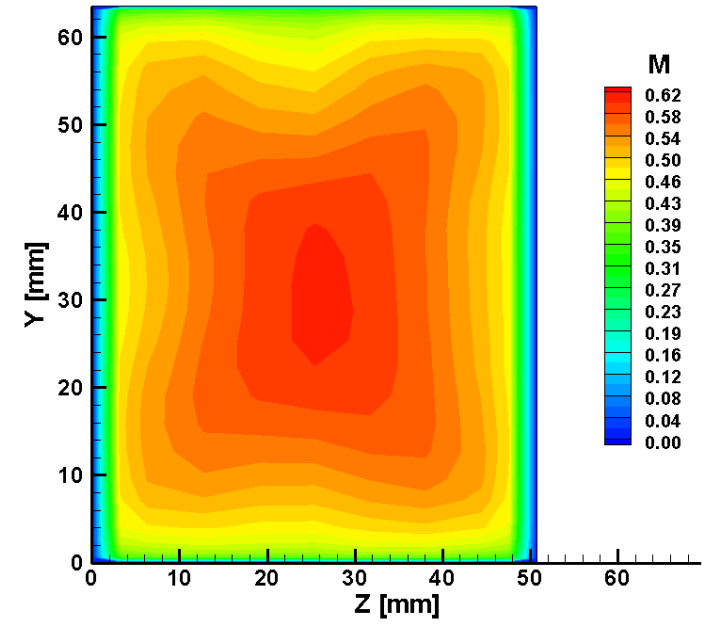

(b) Configuration 3

Figure 8: Mach number contour plots; $M_{\text {set }}=0.5$. 
the lower wall, the boundary layer thickness, $\delta$, decreases from the upstream location (Configuration 2) to the downstream location (Configuration 3), but the displacement thickness, $\delta^{*}$, and momentum thickness, $\theta$, hold virtually constant. Along the upper wall, the boundary layer, displacement and momentum thicknesses increase as the flow travels through the duct. It is not clear whether the differences measured along the upper and lower walls of the hardwall duct are due to measurement resolution issues, or are truly representative of the flow profiles achieved in the duct. When time permits, more detailed repeatability tests will be completed to further evaluate this issue. In addition, a full computation fluid dynamics evaluation is planned to achieve a more detailed understanding of the boundary layer growth through the GFIT. Results of these more detailed flow field studies will be presented in future publications.

\begin{tabular}{|c|c|c|c|c|c|c|c|}
\cline { 3 - 8 } \multicolumn{2}{c|}{} & \multicolumn{3}{c|}{ Lower wall } & \multicolumn{3}{c|}{ Upper wall } \\
\hline Configuration & $M_{\text {set }}$ & $\delta, \mathrm{mm}$ & $\delta^{*}, \mathrm{~mm}$ & $\theta, \mathrm{mm}$ & $\delta, \mathrm{mm}$ & $\delta^{*}, \mathrm{~mm}$ & $\theta, \mathrm{mm}$ \\
\hline \hline 2 & 0.3 & 26.9 & 3.0 & 2.3 & 20.0 & 2.4 & 1.9 \\
\hline 2 & 0.5 & 24.3 & 2.8 & 2.2 & 21.2 & 2.6 & 2.0 \\
\hline 3 & 0.3 & 23.4 & 3.1 & 2.3 & 24.4 & 3.7 & 2.7 \\
\hline 3 & 0.5 & 22.7 & 3.0 & 2.3 & 24.9 & 3.9 & 2.9 \\
\hline
\end{tabular}

Table 1: Flow profile parameters, measured with hardwall insert.

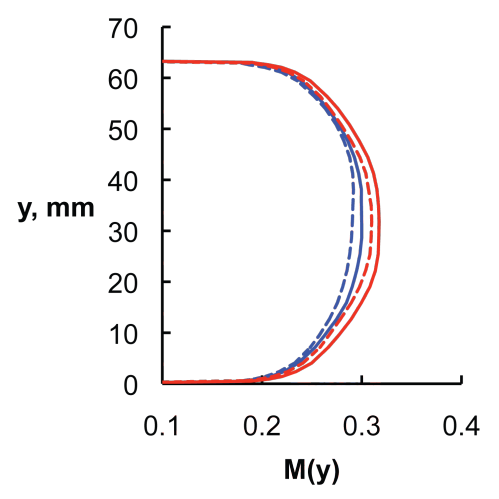

(a) CT57 liner

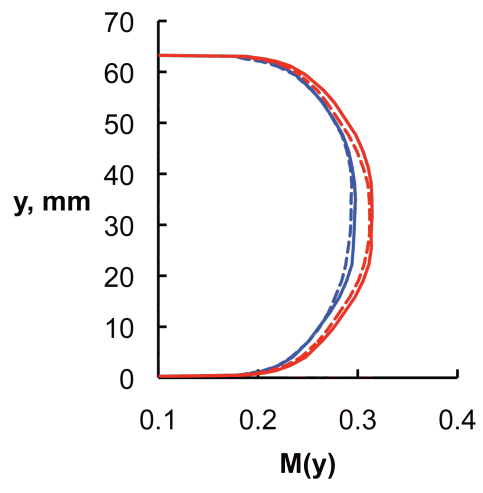

(b) GE01 liner

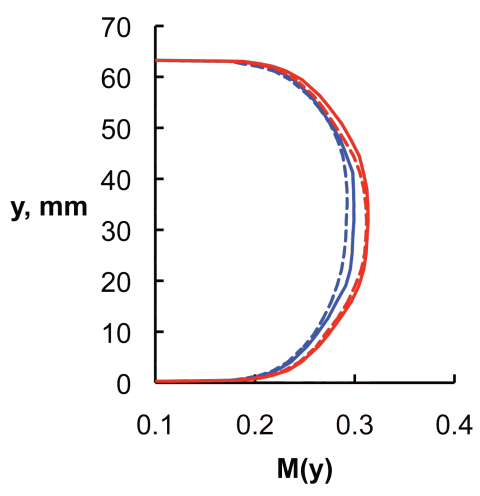

(c) GE04 liner

Figure 9: Flow profiles, $M_{\text {set }}=0.3$. Blue solid and dashed lines - Configurations 1 and 2, respectively; Red solid and dashed lines - Configurations 3 and 4, respectively.

Midspan, 1-D flow profiles were also acquired with each liner installed in the GFIT. Figures 9 and 10 contain 1-D flow profiles acquired with each liner, for centerline, setpoint Mach numbers of 0.3 and 0.5, respectively. In each graph, the blue lines represent data acquired with the test section at the upstream end of the GFIT (solid and dashed lines for Configurations 1 and 2, respectively), while the red lines represent data acquired with the test section at the downstream end of the GFIT (solid and dashed lines for Configurations 3 and 4, respectively). Close inspection reveals minor differences between the effects of the different liners on the flow profiles, but no major features are evident. For the acoustic analysis presented later in this section, these 1-D flow profiles are assumed to vary linearly along the length of the duct (i.e., from the blue dashed line to the blue solid line when the liner is mounted in Configuration 1, or for the corresponding red lines when the liner is mounted in Configuration 3). Hence, the flow profiles used in the shear-flow analysis are essentially a prorated (according to their relative distances from the axial centerpoint of the liner) average of the two profiles acquired for the selected configuration.

\section{Acoustics}

The purpose of the current investigation was to evaluate the effects of mean flow profile (uniform or 1-D shear flow) on the impedance eduction processes used at NASA. The following provides a synopsis of the 


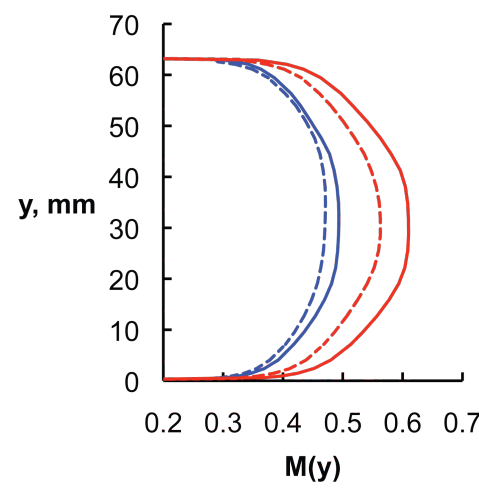

(a) CT57 liner

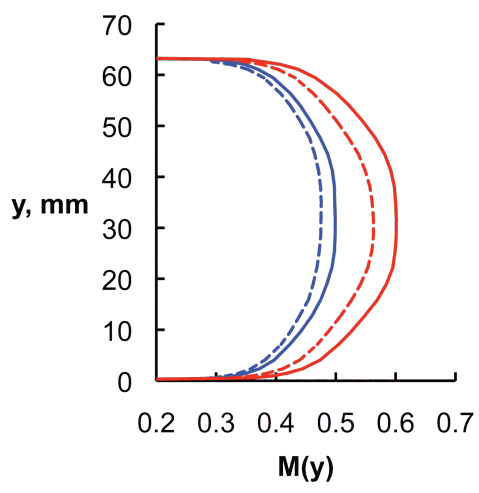

(b) GE01 liner

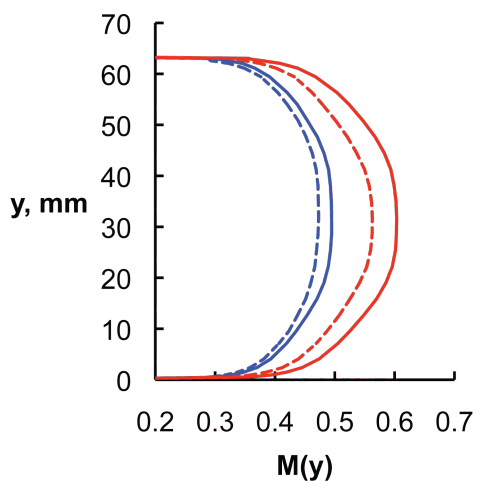

(c) GE04 liner

Figure 10: Flow profiles, $M_{\text {set }}=0.5$. Blue solid and dashed lines - Configurations 1 and 2, respectively; Red solid and dashed lines - Configurations 3 and 4, respectively.

manner in which the selected test liners were intended to be used for this investigation:

1. Evaluate data acquired with the CT57 liner. This liner is expected to be sufficiently linear to be unaffected by source SPL, grazing flow Mach number, or mean flow profile. If this premise is correct, nearly identical results should be achieved regardless of the propagation model used in the impedance eduction process. For the same reasons, the results should also be independent of the axial location of the CT57 liner (the centerline Mach number increases and the corresponding flow profile varies along the length of the duct due to developing boundary layer flow). If these criteria are satisfied, the 1-D shear flow model (PBS-SM) is assumed to be valid (at least for this type of liner).

2. Use the uniform-flow models (CHE-FM and PBU-SM) to evaluate each conventional liner (GE01 and GE04). Based on previous experience, it is expected that their respective resistance spectra will increase with increasing source SPL or grazing flow Mach number, whether the liner is mounted at the upstream or downstream axial location in the GFIT. Their reactance spectra are also expected to follow a $-\cot (k d)$ pattern, where $k$ is the free-space wavenumber and $d$ is the depth of the liner cavity.

3. Use the shear-flow model (PBS-SM) to evaluate each conventional liner, first with the liner mounted in the upstream location, then with it moved to the downstream location. Given the differences in the flow profiles at each of these locations, the effects of these flow profiles on the impedance spectra educed with the PBS-SM can be used to at least partially evaluate the effects of mean flow profile on conventional liners.

The following describes a portion of the results acquired with these liners mounted in the GFIT. As will be shown, the impedances educed with the uniform-flow propagation models follow expected trends, but those educed with the shear-flow propagation model do not. This is true not only for the conventional liners (which might have been expected, given their nonlinearity), but also for the ceramic tubular liner. This indicates further evaluation is needed to better understand the underlying physics. In the meantime, an ad-hoc approach is used to gain insight into the basis for the failure of the 1-D shear-flow model to provide educed impedances that follow expected trends.

\section{Ceramic Tubular Liner (CT57)}

Figure 11 provides the acoustic impedance spectra educed using the PBU-SM at three source sound pressure levels (130, 140 and $150 \mathrm{~dB}$, measured at the source plane, $L_{1}$, in Fig. 1) with the CT57 liner mounted in Configuration 1 (upstream end of GFIT). For this initial case, the mean flow was turned off. As expected, the normalized resistance increases for frequencies near anti-resonance and is relatively constant for frequencies away from anti-resonance. The normalized reactance follows a $-\cot (k d)$ pattern. The effects of source 


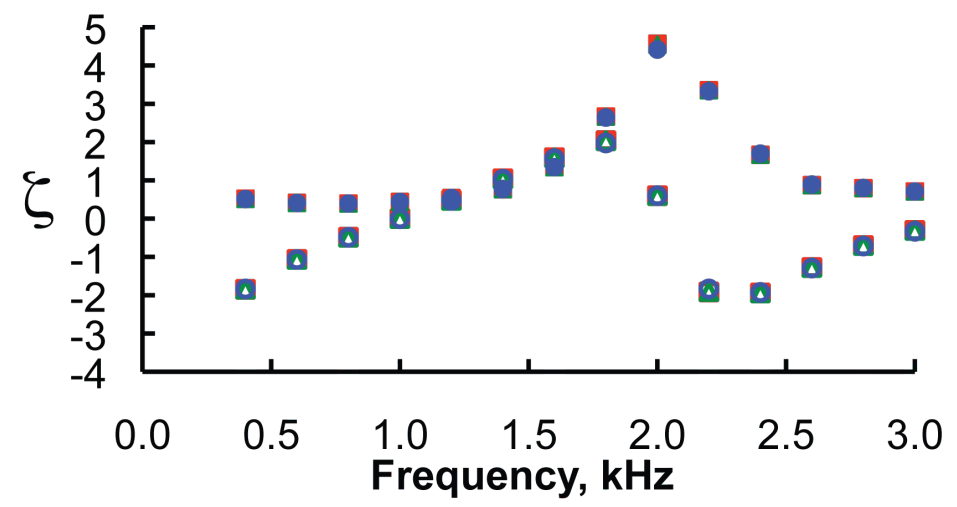

Figure 11: Effects of source SPL on the acoustic impedance spectra educed with the PBU-SM for the CT57 liner positioned in Configuration $1 ; M_{\text {set }}=0.0$. Red squares - $130 \mathrm{~dB}$ source, green triangles - $140 \mathrm{~dB}$ source, blue circles - $150 \mathrm{~dB}$ source; Solid symbols - normalized resistance, open symbols - normalized reactance (All impedances in this paper are normalized by the characteristic impedance of air, $\rho c$, where $\rho$ and $c$ are the density and sound speed in air, respectively).

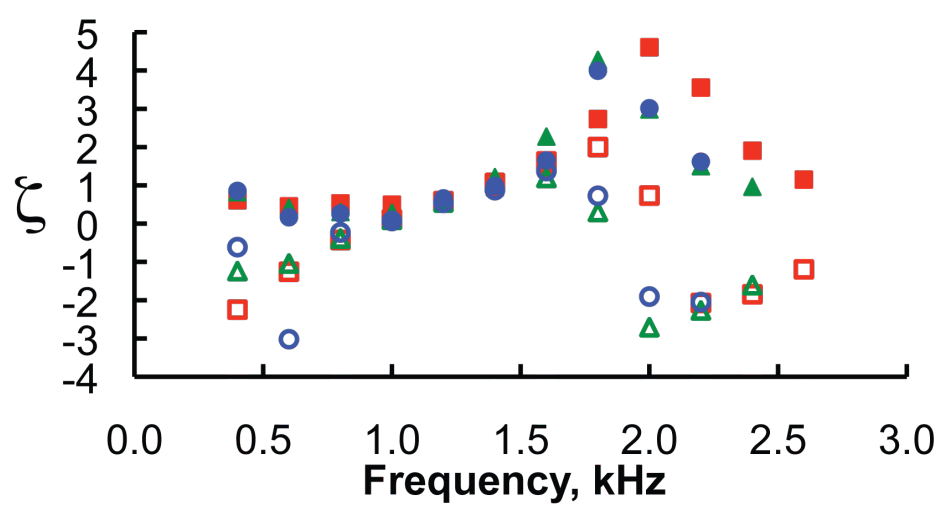

(a) Configuration 1

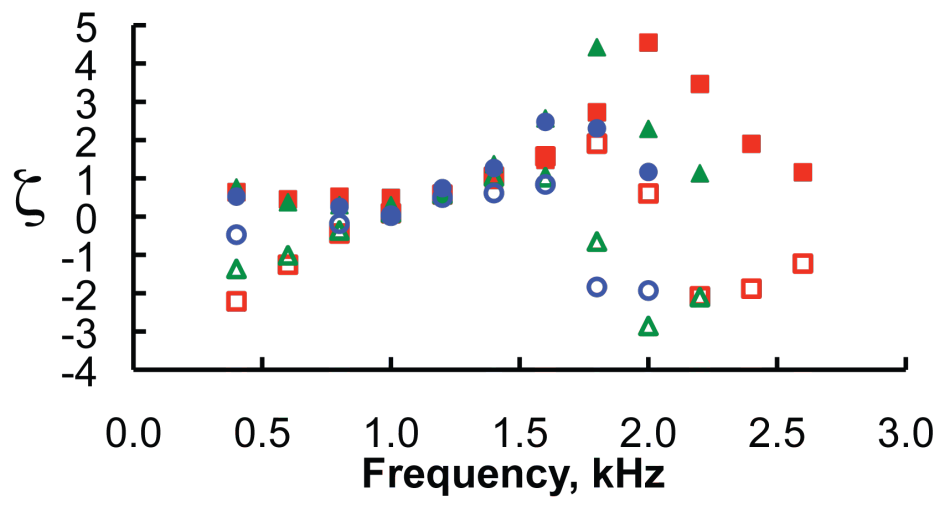

(b) Configuration 3

Figure 12: Effects of Mach number on the acoustic impedance spectra educed with the CHE-FE for the CT57 liner positioned in Configurations 1 and 3, respectively; source SPL of $130 \mathrm{~dB}$. Red squares $-M_{\text {set }}=0.0$, green triangles $-M_{\text {set }}=0.3$, blue circles $-M_{\text {set }}=0.5$; Solid symbols - normalized resistance, open symbols - normalized reactance. 
SPL are imperceptible (symbols for each SPL are overlayed, rendering them indistinguishable), confirming that this liner is linear with respect to SPL. Virtually identical results were educed using the CHE-FE at frequencies below approximately $2.7 \mathrm{kHz}$, for which only plane waves are cut on in the rigid duct. Linearity with respect to SPL was also observed in the presence of mean grazing flow for this liner.

The effects of Mach number on the impedance spectra educed with the finite element method (CHE-FE) are provided in Figure 12, first with the liner postioned in Configuration 1 (part a), then in Configuration 3 (part b). These data were acquired with a source SPL of $130 \mathrm{~dB}$, and provide a number of interesting features. For frequencies away from anti-resonance (e.g., 0.6 to $1.2 \mathrm{kHz}$ ), the effects of Mach number are minimal, suggesting this liner is linear with respect to grazing flow velocity. Also, the anti-resonance is observed to occur at decreased frequency as the Mach number is increased. This is represented by a shift of the peak resistance to lower frequencies as the Mach number increases, which coincides with the transition of the reactance from large positive values to large negative values. At the low frequency end of the spectrum, the resistance increases slightly and the reactance approaches a large negative value, indicating $0.4 \mathrm{kHz}$ is near a low-frequency anti-resonance.

These data were educed using the finite element method to solve the convected Helmholtz equation (CHEFE). Hence, the only differences between the data acquired in Configurations 1 and 3 are the uniform-flow Mach numbers used in the analysis. Given the increase in Mach number as the flow propagates through the duct (see Figs. 9 and 10), the similary between these results (Fig. 12, 'a' and 'b') suggests that Mach number effects are small for the CT57 liner (aside from those near anti-resonance).

The acoustic pressure data were also used with the single-mode method to educe impedance spectra for the CT57 liner in the presence of grazing flow. Figure 13 provides impedance spectra educed with the PBU-SM (red squares) and PBS-SM (green triangles). (An SPL of $140 \mathrm{~dB}$ was chosen for this figure because the $130 \mathrm{~dB}$ data at $M_{\text {set }}=0.5$ was corrupted during file transfers.) The uniform-flow results (PBU-SM) maintain the expected impedance spectrum, and are unaffected by the increase in Mach number. The shearflow results (PBS-SM) are unexpected. For frequencies below resonance, the uniform-flow and shear-flow results follow similar trends. However, for frequencies above resonance, the PBS-SM resistance spectrum is much lower than the PBU-SM resistance spectrum. Also, the reactance spectrum educed with the shear-flow assumption suggests the anti-resonance occurs at a higher frequency than that observed for the uniform-flow assumption. It is also interesting to note that the impedance spectra are nearly identical for the two setpoint Mach numbers for both the PBU-SM and the PBS-SM.

\section{Conventional Liners (GE01 and GE04)}

As indicated earlier, the conventional liners (GE01 and GE04) are nonlinear, i.e., their acoustic resistance spectra increase with source SPL and grazing flow Mach number. Figure 14 provides the effects of source SPL on the acoustic impedance spectra for the GE01 liner. The liner is positioned in Configuration 1 (upstream end of GFIT), and the flow is disengaged. On this scale (all acoustic impedance spectra are plotted on the same scale for ease of comparison), the effects of source SPL appear small. The normalized resistance increases slightly near resonance (positive-going zero crossing of the reactance), but is relatively constant at approximately 0.1 to 0.2 across most of the frequency range. At first glance, this appears remarkably low. However, the reader is reminded that this is a conventional liner, designed for usage in an aircraft engine nacelle. Hence, its target flow regime is approximately Mach 0.3 to 0.5. Thus, since the liner is nonlinear (i.e., resistance increases with flow velocity), it is designed to have a low resistance at Mach 0.0, but a reasonable resistance (nearer 1 to 2 ) at Mach 0.3 to 0.5 . These data were educed using the finite element method (CHE-FE), but are also representative of those achieved with the single-mode method (PBU-SM).

Figure 15 shows the effects of Mach number on the GE01 acoustic impedance, with the liner mounted at the upstream (Configuration 1) and downstream (Configuration 3) ends of the GFIT. These impedance spectra were educed using the finite element method (CHE-FE). At Mach 0.0 (red squares, solid for resistance and open for reactance), the impedance spectrum is as expected, as discussed earlier. At Mach 0.3 (green triangles), the normalized resistance increases to approximately 0.75 , and the reactance starts to "flatten out," especially at the higher frequencies above resonance. The reactance also rises noticeably at $0.4 \mathrm{kHz}$. As discussed earlier, this suggests that this frequency approaches a low frequency anti-resonance. At Mach 0.5 (blue circles), the normalized resistance increases further, to approximately 1.0, while the reactance flattens out even more above resonance. 


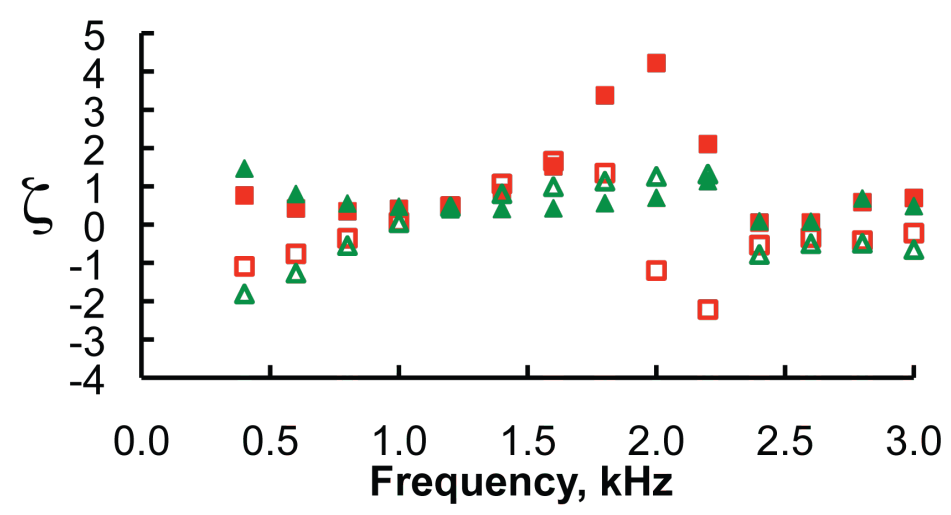

(a) $M_{\text {set }}=0.3$

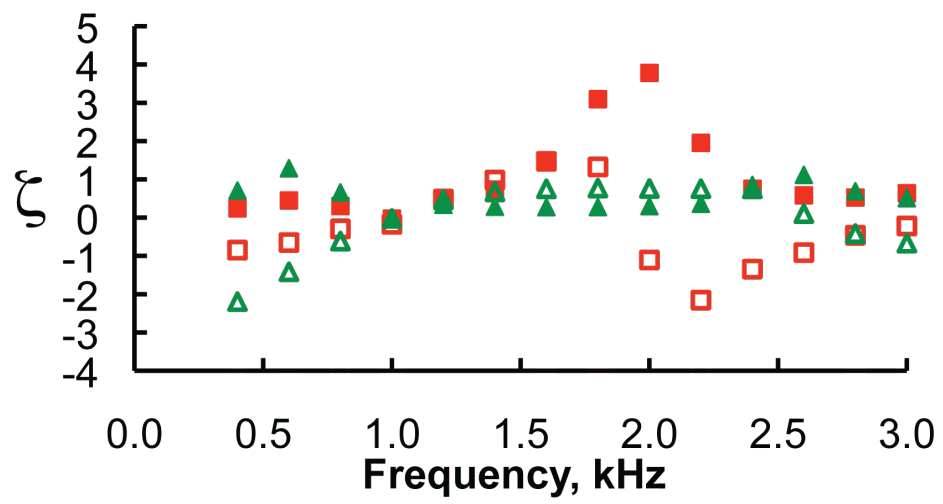

(b) $M_{\text {set }}=0.5$

Figure 13: Effects of mean flow model on the acoustic impedance spectra educed with the single-mode method for the CT57 liner positioned in Configuration 1; source SPL of $140 \mathrm{~dB}$. Red squares - uniform flow assumption (PBU-SM), green triangles - 1-D shear flow assumption (PBS-SM); Solid symbols - normalized resistance, open symbols - normalized reactance.

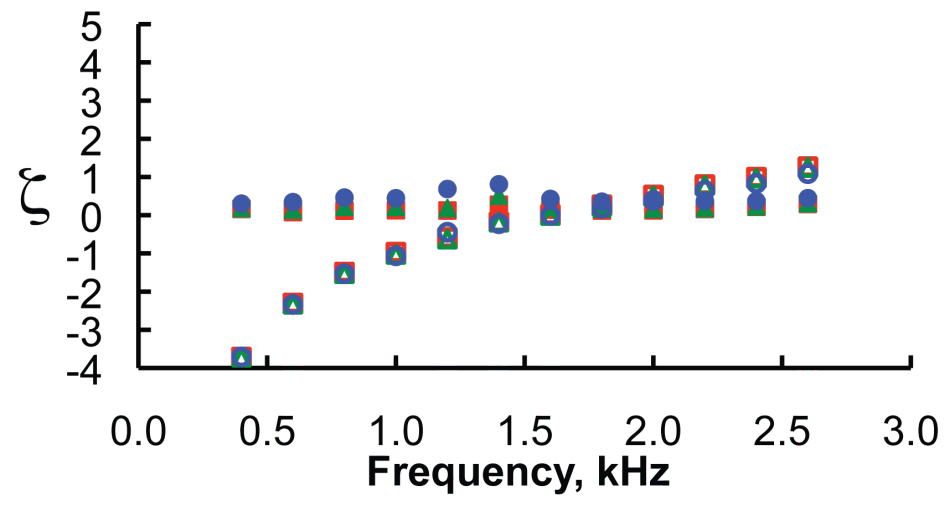

Figure 14: Effects of source SPL on the acoustic impedance spectra educed with the finite element method (CHE-FE) for the GE01 liner positioned in Configuration 1, $M_{\text {set }}=0.0$. Red squares - $130 \mathrm{~dB}$ source, green triangles - $140 \mathrm{~dB}$ source, blue circles - $150 \mathrm{~dB}$ source; Solid symbols - normalized resistance, open symbols - normalized reactance. 


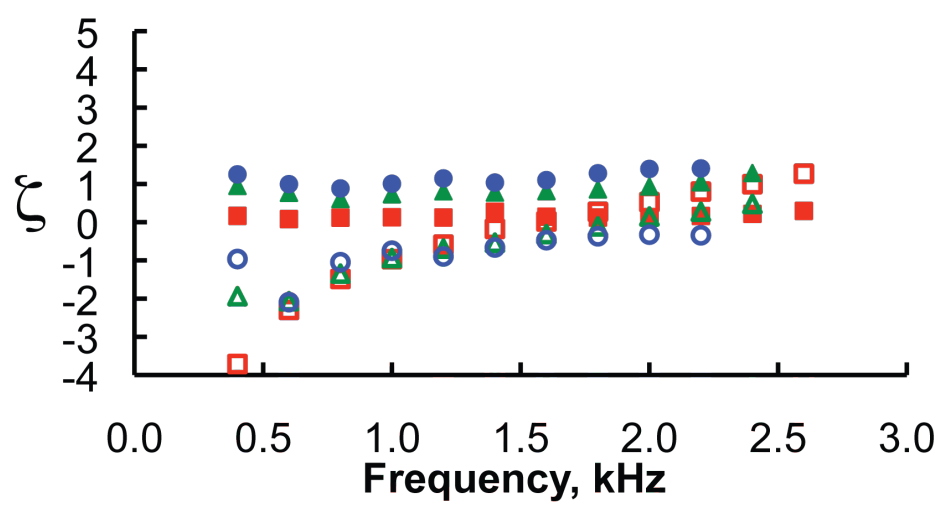

(a) Configuration 1

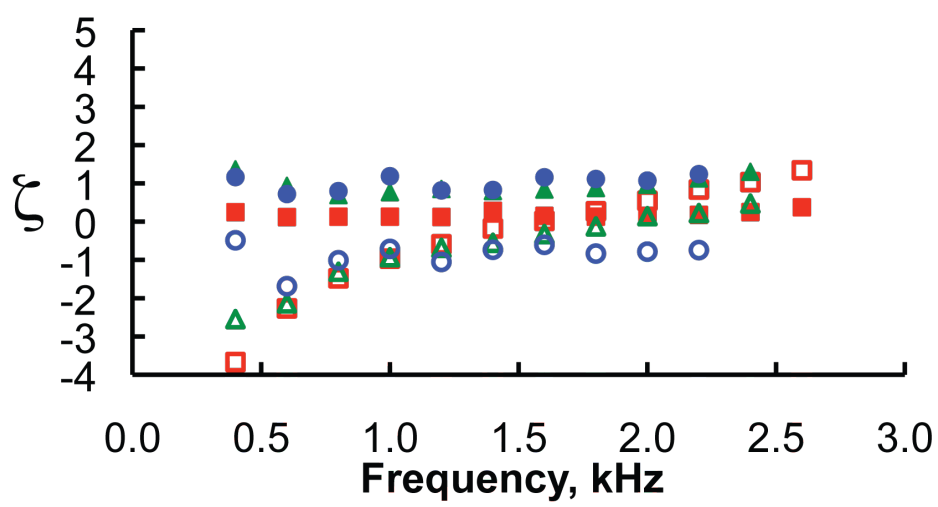

(b) Configuration 3

Figure 15: Effects of Mach number on the acoustic impedance spectra educed with the finite element method (CHE-FE) for the GE01 liner positioned in Configurations 1 and 3, respectively; source SPL of $130 \mathrm{~dB}$. Red squares $-M_{\text {set }}=0.0$, green triangles $-M_{\text {set }}=0.3$, blue circles $-M_{\text {set }}=0.5$; Solid symbols - normalized resistance, open symbols - normalized reactance. 


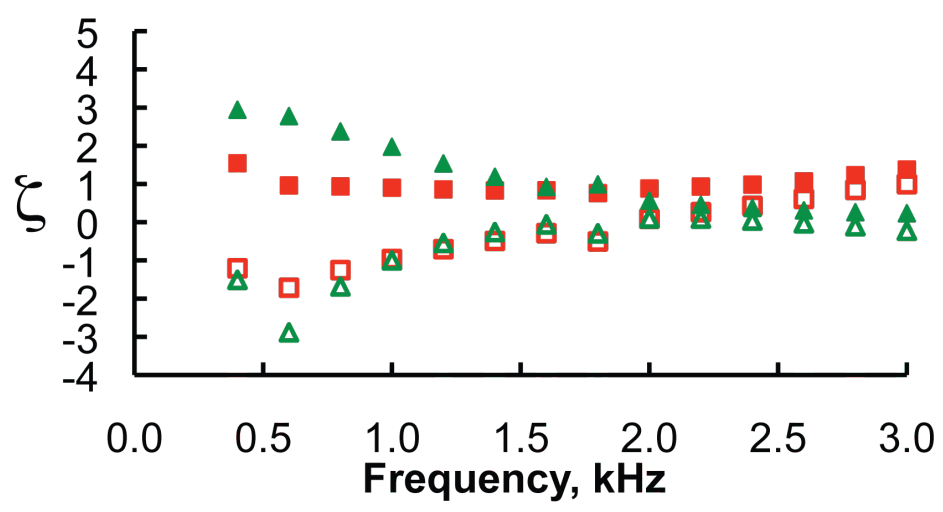

(a) $M_{\text {set }}=0.3$

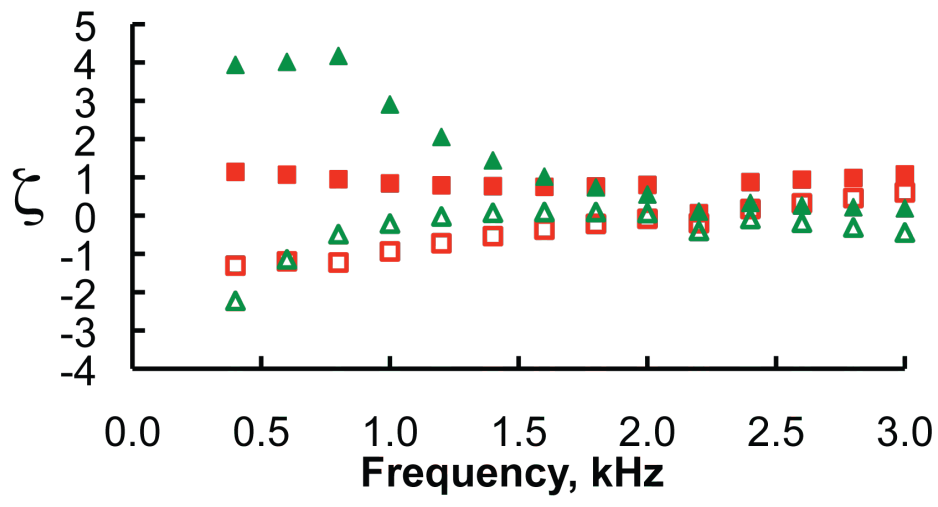

(b) $M_{\text {set }}=0.5$

Figure 16: Effects of mean flow model on the acoustic impedance spectra educed with single-mode method for the GE01 liner positioned in Configuration 1; source SPL of $130 \mathrm{~dB}$. Red squares - uniform flow assumption (PBU-SM), green triangles - 1-D shear flow assumption (PBS-SM); Solid symbols - normalized resistance, open symbols - normalized reactance. 


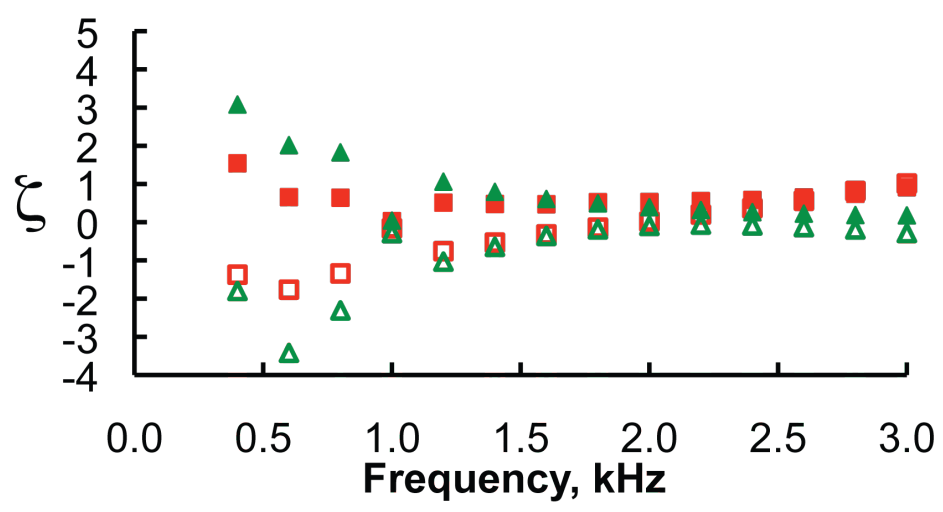

(a) $M_{\text {set }}=0.3$

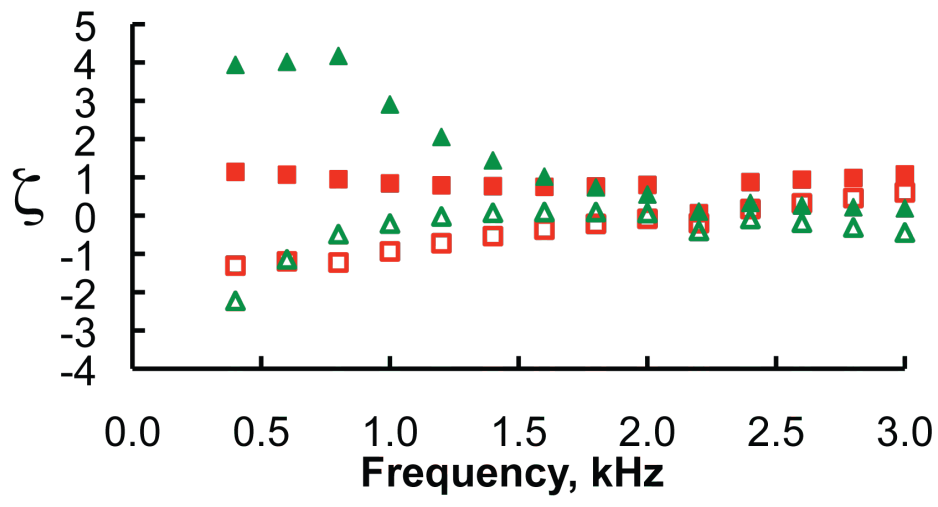

(b) $M_{\text {set }}=0.5$

Figure 17: Effects of mean flow model on the acoustic impedance spectra educed with single-mode method for the GE04 liner positioned in Configuration 1; source SPL of $130 \mathrm{~dB}$. Red squares - uniform flow assumption (PBU-SM), green triangles - 1-D shear flow assumption (PBS-SM); Solid symbols - normalized resistance, open symbols - normalized reactance. 
The single-mode method was also used to educe the impedance spectra for the GE01 liner. Figure 16 provides a direct comparison of single-mode method results achieved with the uniform and 1-D shear flow profiles, for a source SPL of $130 \mathrm{~dB}$. In this figure, the red squares and green triangles depict the impedance spectra educed with uniform (PBU-SM) and 1-D shear-flow (PBS-SM) assumptions, respectively. The uniform-flow results are similar to those educed using the finite-element method (CHE-FE), but the shearflow results are noticeably different. The resistance spectrum is changed from a relatively flat curve to one with a negative slope, with the two curves (resistance with uniform flow and shear flow assumptions) crossing at or near resonance. At a setpoint Mach number of 0.3 , the reactance is relatively independent of the flow assumption for frequencies below resonance, but is significantly altered for frequencies above resonance. As the Mach number is increased further, these effects of flow assumption are magnified further. In fact, the reactance below resonance actually changes sign. Figure 17 demonstrates that these effects are also evident for the GE04 liner as well.

\section{Investigation of 1-D Shear-Flow Model}

The results presented above indicate the shear-flow model used in the current analysis was insufficient. Two potential causes have been identified to explain the failure of this model.

1. The model contains insufficient fidelity to represent the physics of the problem.

2. The measured data is of insufficient resolution for use with the 1-D shear-flow model.

Clearly, a 3-D shear-flow model is needed to fully capture the effects of developing boundary layer flow present in the GFIT. As an intermediate step, the authors intend to implement a propagation model that supports parallel (no change over the axial length of the liner), 2-D shear flow profiles. Results of this extension to the model will be the subject of future reports.

Given the method used to measure the mean flow profiles in the current study (traversing pitot probe), the shape of the profile near the upper and lower walls of the duct causes the greatest concern. A computational fluid dynamics (CFD) analysis is planned to evaluate the mean flow through the GFIT in much greater detail, and this analysis is expected to provide improved 1-D shear-flow profiles (especially near the upper and lower walls) for use in the impedance eduction process. In the meantime, an ad-hoc approach is applied to evaluate how the shape of the flow profile affects the impedances educed with the model (PBS-SM). The shape of the profile $(d M / d y)$ near the wall is modified by simply multiplying the entire flow profile by a constant factor $\left(m_{f a c}\right)$. Clearly, $m_{f a c}$ values greater than unity will increase the Mach number gradient near the wall, but will also increase the Mach number at all other heights ( $y$-direction). This increase of $M_{a v e}$ is not of great concern for evaluation of the CT57 liner, as it is insensitive to grazing flow Mach number. For the conventional liners, the results provided earlier (e.g., Fig. 15) suggest that these liners are weakly nonlinear (i.e., their resistance spectra increase slightly with an increase in $M_{\text {set }}$ from 0.3 to 0.5 ), so the increase in $M_{\text {ave }}$ should not be a dominant factor. Thus, this simple, ad hoc approach is considered acceptable to gain insight into the effects of the flow gradient near the wall.

Figure 18 provides results achieved with the CT57 liner. Parts (a) and (b) of this figure compare impedance spectra educed with the two single-mode methods (PBU-SM and PBS-SM), with the liner mounted in the upstream location (Configuration 1) and a source SPL of $130 \mathrm{~dB}$. If a multiplicative factor of $1.8\left(m_{f a c}=1.8\right)$ is used, the 1-D shear flow results (PBS-SM) are well matched to those achieved with uniform flow (PBU-SM). This holds for both Mach numbers considered in this study. However, if the CT57 liner is moved to the downstream location (Configuration 3, see Fig. 18-(c)), the optimum multiplicative factor decreases slightly $\left(m_{f a c}=1.7\right)$.

Figure 19 provides the corresponding results for the GE01 liner. It is interesting to note that the values for $m_{f a c}$ that provide optimum comparisons (i.e., impedance spectra are nearly the same when educed with either the PBU-SM or the PBS-SM) for the CT57 liner are also suitable for use with the GE01 liner (similar results are achieved with the GE04 liner as well). A factor of 1.8 produces optimum comparisons of impedance spectra educed with the uniform-flow and "modified", 1-D shear-flow models when the test liner is mounted in the upstream location, and the optimized factor reduces to 1.7 when the liner is moved to the downstream location. As before, these results are independent of the grazing flow Mach number $\left(M_{\text {set }}\right)$.

Thus, by "calibrating" the model with the CT57 liner to account for flow profile changes over the length of the GFIT (i.e., $m_{f a c}=1.8$ and $m_{f a c}=1.7$ at upstream and downstream locations, respectively), the 


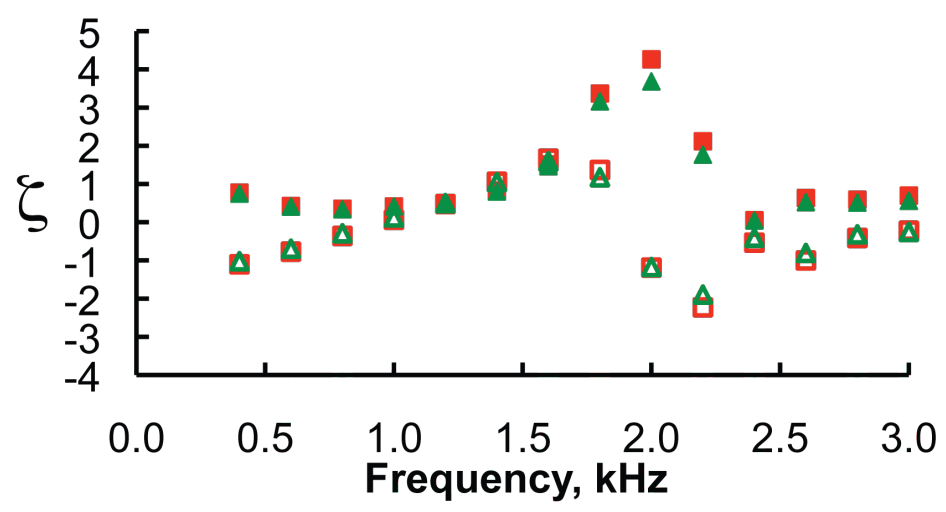

(a) $M_{\text {set }}=0.3$, Configuration $1, m_{f a c}=1.8$

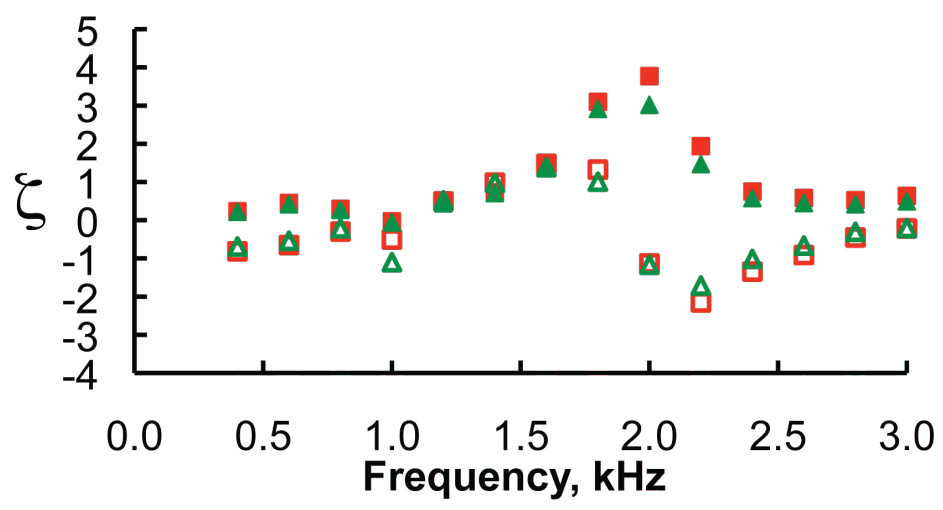

(b) $M_{\text {set }}=0.5$, Configuration $1, m_{f a c}=1.8$

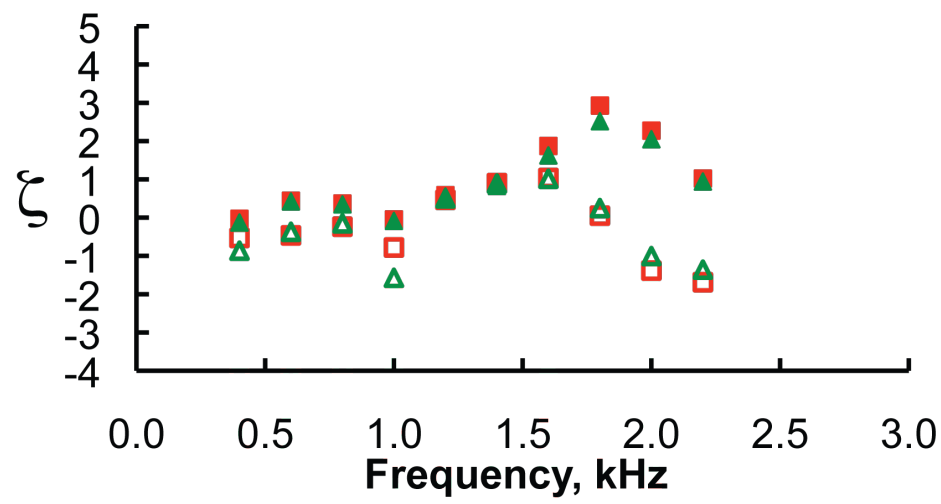

(c) $M_{\text {set }}=0.3$, Configuration $3, m_{f a c}=1.7$

Figure 18: Effects of "modified" mean flow model on the acoustic impedance spectra educed with the singlemode method for the CT57 liner; source SPL of $130 \mathrm{~dB}$. Red squares - uniform flow assumption (PBU-SM), green triangles - 1-D shear flow assumption (PBS-SM); Solid symbols - normalized resistance, open symbols - normalized reactance. 


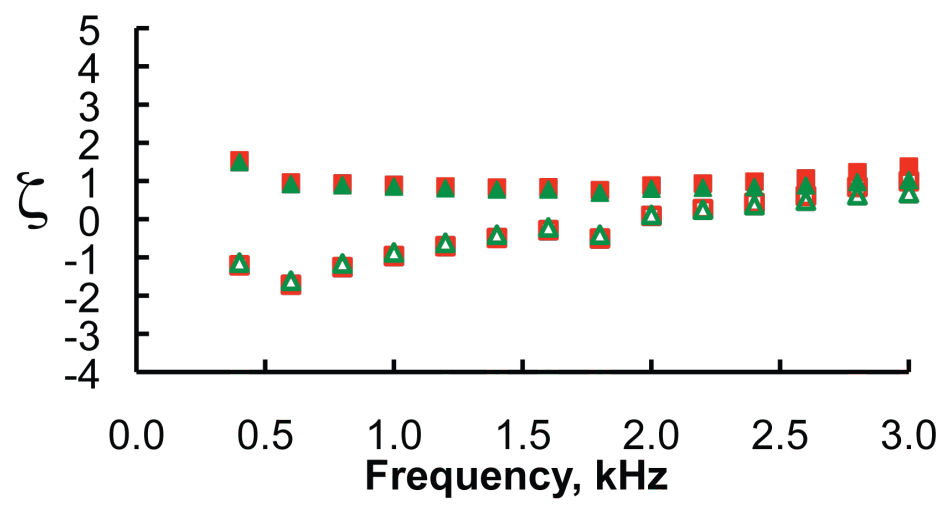

(a) $M_{\text {set }}=0.3$, Configuration $1, m_{f a c}=1.8$

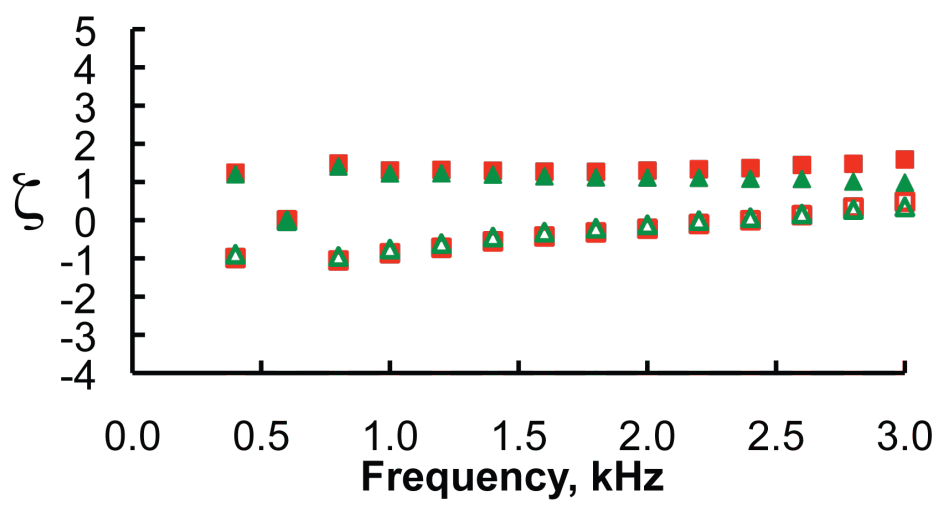

(b) $M_{\text {set }}=0.5$, Configuration $1, m_{f a c}=1.8$

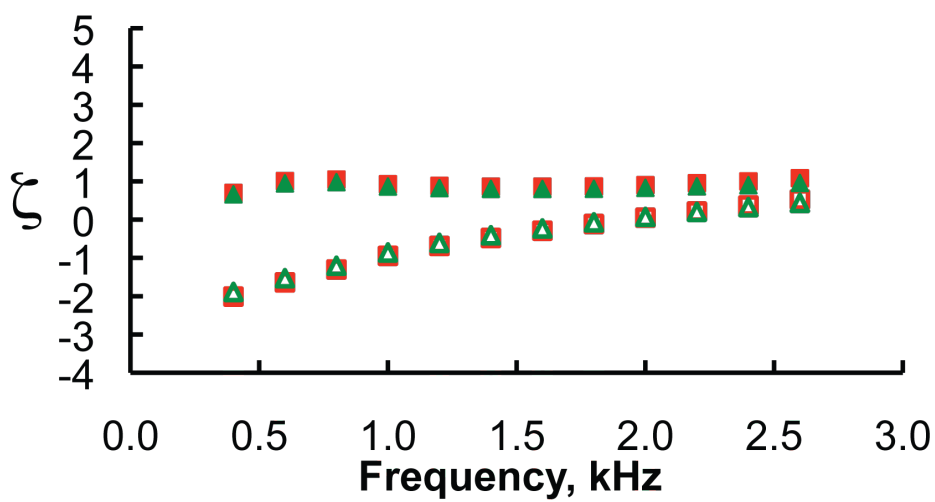

(c) $M_{\text {set }}=0.3$, Configuration $3, m_{f a c}=1.7$

Figure 19: Effects of "modified" mean flow model on the acoustic impedance spectra educed with the singlemode method for the GE01 liner; source SPL of $130 \mathrm{~dB}$. Red squares - uniform flow assumption (PBU-SM), green triangles - 1-D shear flow assumption (PBS-SM); Solid symbols - normalized resistance, open symbols - normalized reactance. 
corresponding results for the conventional liners indicate they are also weakly dependent on the effects of flow profile. However, it should be noted that, although these modifications to the shear-flow profile cause the educed impedance spectra to follow expected trends, this does not suggest that these modified flow profiles are correct. Clearly, the values of $m_{f a c}$ that provide desirable results are well beyond the measurement uncertainty of the data acquisition system, at least for Mach numbers measured across the core of the duct. However, it is certainly possible that resolution of the measured flow data near the walls is off by factors of this magnitude. This will be explored in detail in future studies.

\section{Concluding Remarks}

This paper has presented results of an investigation of the effects of grazing flow on impedance eduction processes employed at NASA Langley. Three acoustic liners were used in this study. One is a ceramic tubular liner designed to be linear with respect to source sound pressure level and grazing flow velocity, while the other two are conventional, perforate-over-honeycomb liners that are weakly nonlinear with respect to each of these parameters. Acoustic tests were conducted at source sound pressure levels of 130, 140 and $150 \mathrm{~dB}$, and at setpoint, centerline Mach numbers of 0.0, 0.3 and 0.5, such that the nonlinearity of each liner could be assessed. Flow profiles acquired at four axial locations in the Langley Grazing Flow Impedance Tube (GFIT) were used as input to a propagation model based on the Pridmore-Brown equation, along with SPL and phase decay rates based on acoustic pressures measured via microphones flush-mounted on the wall opposite the liner, to educe the impedance of each of the three acoustic liners in the presence of grazing flow. This method allows the use of uniform or 1-D shear flow profiles. The acoustic pressure data were also input to a propagation code based on the convected Helmholtz equation that uses a finite element method to educe the acoustic impedance of the liner. This method uses a uniform-flow assumption. Comparisons of the results from these analyses suggest:

1. The ceramic tubular liner is nearly linear with respect to source sound pressure level and grazing flow velocity.

2. The two conventional liners are weakly nonlinear, as indicated by an increase in their resistance spectra with increases in source sound pressure level or grazing flow velocity.

3. The finite element method for solving the convected Helmholtz equation provides impedance spectra that follow expected trends. The resistance increases with increasing source sound pressure level or grazing flow velocity, and the reactance follows a $-\cot (k d)$ pattern.

4. The uniform-flow, single-mode method for solving the Pridmore-Brown equation provides impedance spectra that are consistent with those achieved with the finite element method.

5. The 1-D shear flow version of the single-mode method produces impedance spectra that do not follow expected trends. These spectra tend to match those achieved with the uniform-flow assumption for frequencies at or below resonance, but deviate appreciably for frequencies above resonance.

6. A simple, ad hoc approach, in which the measured 1-D shear-flow profiles are increased by a constant multiplicative factor for use in the single-mode method, provides results consistent with those achieved with a uniform-flow method. These results indicate the effects of flow profile (at least for those included in the current study) on the educed impedance of conventional liners are small. The consistency of these results provides insights that can be used for further evalautions of the shear flow effects on the NASA impedance eduction processes.

Additional studies are planned with the data acquired in the current investigation. The mean flow profiles acquired with the hardwall insert will be evaluated using a CFD analysis to gain further insight into the boundary layer growth through the GFIT. In addition, a propagation model based on the linearized Euler equations will be implemented to use 2-D shear flow profiles derived from this CFD analysis. It is expected that the inclusion of two-dimensional effects into the analysis should improve the ability to educe acoustic impedance spectra of acoustic liners in the presence of grazing flow. 


\section{Acknowledgements}

The authors wish to express their appreciation for the diligent efforts of Martha Brown, Carol Harrison, Brian Howerton, Christal Kellam and Jerry Lyle in the acquisition of the data used in this investigation. This research was funded by the Subsonic Fixed Wing Project of NASA's Fundamental Aeronautics Program.

\section{References}

${ }^{1}$ Pridmore-Brown, D. C., "Sound Propagation in a Fluid Flowing Through an Attenuating Duct," Journal of Fluid Mechanics, Vol. 4, 1958, pp. 393-406.

${ }^{2}$ Tack, D. H. and Lambert, F. F., "Influence of Shear Flow on Sound Attenuation in a Lined Duct," Journal of the Acoustical Society of America, Vol. 38, 1965, pp. 655-666.

${ }^{3}$ Eversman, W., "Effect of Boundary Layer on the Transmission and Attenuation of Sound in an Acoustically Treated Circular Duct," Journal of the Acoustical Society of America, Vol. 49, No. 5, 1971, pp. 1372-1380.

${ }^{4} \mathrm{Li}$, X. D., Richter, C., and Thiele, F., "Time-Domain Impedance Boundary Conditions for Surfaces with Subsonic Mean Flows," Journal of the Acoustical Society of America, Vol. 119, No. 5, May 2006, pp. 2665-2676.

${ }^{5}$ Watson, W. R., Tracy, M. B., Jones, M. G., and Parrott, T. L., "Impedance Eduction in the Presence of Shear Flow," AIAA Paper 2001-2263, May 2001.

${ }^{6}$ Watson, W. R., Jones, M. G., and Parrott, T. L., "Validation of an Impedance Eduction Method in Flow," AIAA Journal, Vol. 37, No. 7, June 1998, pp. 818-824.

${ }^{7}$ Jones, M. G., Watson, W. R., Tracy, M. B., and Parrott, T. L., "Comparison of Two Waveguide Methods for Educing Liner Impedances in Grazing Flow," AIAA Journal, Vol. 42, No. 2, Februrary 2004, pp. 232-240.

${ }^{8}$ Jones, M. G., Watson, W. R., and Parrott, T. L., "Benchmark Data for Evaluation of Aeroacoustic Propagation Codes with Grazing Flow," AIAA Paper 2005-2853, May 2005.

${ }^{9}$ Watson, W., Jones, M., and Parrott, T., "A Quasi-3-D Theory for Impedance Eduction in Uniform Grazing Flow," AIAA Paper 2005-2848, May 2005.

${ }^{10}$ Watson, W. R. and Jones, M. G., "Comparison of Convected Helmholtz and Euler Model for Impedance Eduction in Flow," AIAA Paper 2006-2643, May 2006.

${ }^{11}$ Jones, M. G., Watson, W. R., and Nark, D. M., "Optimization of Acoustic Pressure Measurements for Impedance Eduction," AIAA Paper 2007-3531, May 2007.

${ }^{12}$ Mani, R., Luedke, J., Jones, M. G., and Nark, D. M., "Improved Inlet Noise Attenuation by Alteration of Boundary Layer Profiles," ACTIVE Paper 2004-87, September 2004.

${ }^{13}$ Jones, M. G., Parrott, T. L., and Watson, W. R., "Comparison of Acoustic Impedance Eduction Techniques for LocallyReacting Liners," AIAA Paper 2003-3306, May 2003.

${ }^{14}$ Watson, W. R. and Jones, M. G., "Impedance Eduction in Ducts with Higher-Order Modes and Flow," AIAA Paper 2009-3236, May 2009.

${ }^{15}$ Kraft, R. E., "Theory and Measurement of Acoustic Wave Propagation in Multi-Sectioned Rectangular Ducts," Ph.d. dissertation, university of cincinnati, cincinnati, oh, May 1976.

${ }^{16}$ Myers, M. K., "On the Acoustic Boundary Condition in the Presence of Flow," Journal of Sound and Vibration, Vol. 71, No. 3, 1980, pp. 429-434.

${ }^{17}$ Parrott, T. L. and Jones, M. G., "Parallel-Element Liner Impedances for Improved Absorption of Broadband Sound in Ducts," Noise Control Engineering Journal, Vol. 43, No. 6, November - December, 1995.

${ }^{18}$ Bendat, J. S. and Piersol, A. G., Random Data: Analysis and Measurement Procedures, Wiley-Interscience, 1971. 